\title{
Augmented Method to Improve Thermal Data for the Figure Drift Thermal Distortion Predictions of the JWST OTIS Cryogenic Vacuum Test
}

\author{
Sang C. Park ${ }^{* a}$, Timothy M. Carnahan ${ }^{b}$, Lester M. Cohen ${ }^{\mathrm{a}}$, Cherie B. Congedo ${ }^{\mathrm{e}}$, Michael J. Eisenhower ${ }^{\mathrm{a}}$, \\ Wes Ousley ${ }^{\mathrm{d}}$, Andrew Weaver ${ }^{\mathrm{c}}$, Kan Yang ${ }^{\mathrm{b}}$ \\ a. Smithsonian Astrophysical Observatory, Cambridge, MA 02140 USA \\ ${ }^{b .}$ NASA Goddard Space Flight Center, Greenbelt MD 20771 USA \\ c. ATA Aerospace., Greenbelt MD 20770 USA \\ d. Genesis Engineering Solutions LLC, Lanham, MD 20706 \\ ${ }^{e}$ SGT, Inc., Greenbelt MD 20770 USA
}

\begin{abstract}
The JWST Optical Telescope Element (OTE) assembly is the largest optically stable infrared-optimized telescope currently being manufactured and assembled, and is scheduled for launch in 2018. The JWST OTE, including the 18 segment primary mirror, secondary mirror, and the Aft Optics Subsystem (AOS) are designed to be passively cooled and operate near $45 \mathrm{~K}$. These optical elements are supported by a complex composite backplane structure. As a part of the structural distortion model validation efforts, a series of tests are planned during the cryogenic vacuum test of the fully integrated flight hardware at NASA JSC Chamber A. The successful ends to the thermal-distortion phases are heavily dependent on the accurate temperature knowledge of the OTE structural members. However, the current temperature sensor allocations during the cryo-vac test may not have-sufficient fidelity to provide accurate knowledge of the temperature distributions within the composite structure. A method based on an inverse distance relationship among the sensors and thermal model nodes was developed to improve the thermal data provided for the nanometer scale WaveFront Error (WFE) predictions. The Linear Distance Weighted Interpolation (LDWI) method was developed to augment the thermal model predictions based on the sparse sensor information. This paper will encompass the development of the LDWI method using the test data from the earlier 'pathfinder' cryo-vac tests, and the results of the notional and as tested WFE predictions from the structural finite element model cases to characterize the accuracies of this LDWI method.
\end{abstract}

Keywords: JWST, Thermal Distortion, nanometer, Composite, Model Validation, Thermal Modeling, FEM, Cryogenic, Vacuum Test

\section{NOMENCLATURE}

\begin{tabular}{|c|c|}
\hline $\mathrm{AD}$ & $=$ Alignment Drift \\
\hline AOS & $=$ Aft Optics Subsystem \\
\hline $\mathrm{CS}$ & $=$ Center Section \\
\hline $\mathrm{CV}$ & $=$ Cryo Vacuum \\
\hline DoF & $=$ degree of freedoms \\
\hline FD & $=$ Figure Drift \\
\hline FEM & $=$ Finite Element Model \\
\hline $\mathrm{GHe}$ & $=$ Gaseous Helium \\
\hline GSE & $=$ Ground Support Equipment \\
\hline GSFC & $=$ NASA Goddard Space Flight Center \\
\hline ISIM & $=$ Integrated Science Instrument Module \\
\hline
\end{tabular}

Sang C. Park*sapark@cfa.harvard.edu*www.cfa.harvard.edu 


\begin{tabular}{|c|c|}
\hline JSC & $=$ NASA Johnson Space Center \\
\hline JWST & $=$ James Webb Space Telescope \\
\hline $\mathrm{K}$ & $=$ Kelvin (unit of Temperature) \\
\hline LDWI & $=$ Linear Distance Weighted Interpolation \\
\hline $\mathrm{mK}$ & $=$ milli-Kelvin (unit of Temperature) \\
\hline MUF & $=$ Model Uncertainty Factor \\
\hline MWIF & $=$ MultiWave Interferometer \\
\hline NASA & $=$ National Aeronautics and Space Administration \\
\hline NGST & $=$ Northrop Grumman Space Technology \\
\hline $\mathrm{nm}$ & $=$ nano-meter, $10^{-9}$ meter \\
\hline OGSE & $=$ Optical Ground Support Equipment \\
\hline OTE & $=$ Optical Telescope Element \\
\hline OTIS & $=\mathrm{OTE}$ and ISIM Assembly \\
\hline PMBSS & $=$ Primary Mirror Backplane Support Structure \\
\hline $\mathrm{PF}$ & $=$ PathFinder \\
\hline PMSA & $=$ Primary Mirror Segment Assembly \\
\hline PM & $=$ Primary Mirror \\
\hline $\mathrm{SAO}$ & $=$ Smithsonian Astrophysical Observatory \\
\hline SM & $=$ Secondary Mirror \\
\hline SMA & $=$ Secondary Mirror Assembly \\
\hline STOP & $=$ Structural Thermal Optical Analysis \\
\hline UUT & $=$ Unit Under Test \\
\hline
\end{tabular}

\section{INTRODUCTION}

The primary objective of the James Webb Space Telescope (JWST) mission is to observe the early universe at a time when the first stars and galaxies were beginning to form. Outfitted with a light-weighted beryllium cryogenic 18segment, 6.5 meter primary mirror and a complement of near- and mid-infrared sensing cameras and spectrometers, JWST will allow astronomers to study the universe as it emerged from the dark ages that followed the Big Bang. In addition, the telescope provides the unique capability to study the evolution of galaxies, the history of the Milky Way, and the origin and formation of planetary systems.

As an international collaboration among the space agencies of the US, Europe, and Canada, JWST is scheduled to be launched in 2018 by an Ariane 5 launch vehicle from Korou, French Guiana. After a six-month journey, JWST will enter orbit around the Earth-Sun L2 Lagrange point, about 1.5 million km from the Earth in the anti-Sun direction. In this orbit, the telescope and instruments will be shadowed from Earth and Sun inputs by the large deployable sunshield, allowing passive cooling to cryogenic temperatures (see Figure 1). JWST mission is optimized for infrared wavelengths $(0.6-28.5$ microns) therefore, the telescope elements must be able to achieve the operational temperatures below $54 \mathrm{~K}$. This range of wavelengths will enable scientists to peer into dust clouds where stars and planetary systems are, and extend discoveries beyond the current

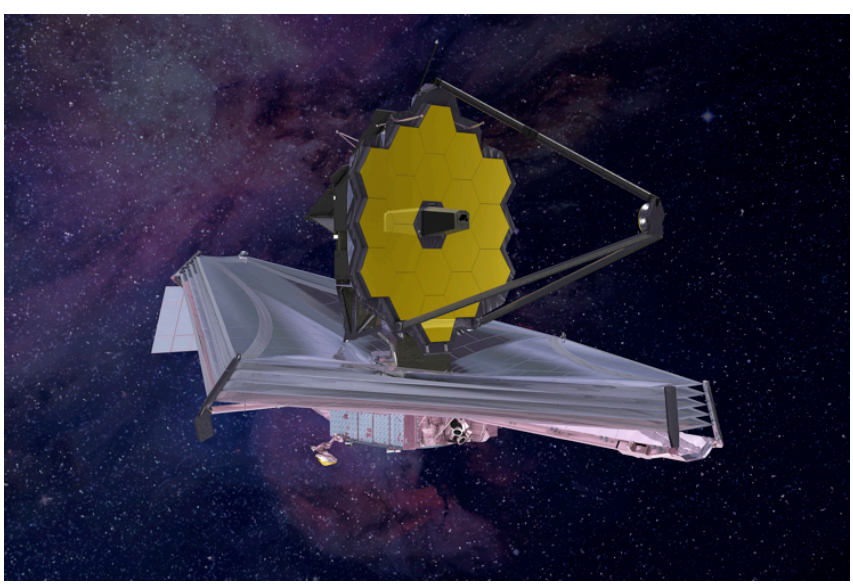

Figure 1 On-orbit Deployed Configuration of JWST capabilities of Hubble Space Telescope. Due to the size of the sunshield and the cold temperature of the optical system, 
the deployed JWST cannot be thermally or optically tested at the combined 'observatory' level in any facilities that currently exist. Instead, sub-assemblies will be optically and thermally test-verified, and analysis will be used to show compliance with system requirements, including margins.

\section{THERMAL DISTORTION MODELING AND TESTING OF THE JWST}

Once on-orbit, as JWST re-orients, or slews, to new points in the sky there will be associated changes in the thermal state of the telescope. These thermal changes will cause changes in the telescope performance. When the performance has been sufficiently degraded, the Primary Mirror (PM) and Secondary Mirror (SM) will need to be readjusted. This is possible because JWST's PM has 18 actuated segments, the first time NASA implemented such PM / SM actuation mechanisms. However, refocusing the telescope takes away from valuable science observation time. In order to understand the limits of the telescope range while changing orientations, a thermal model was used to predict the temperature changes from one state to another. The predictions are then applied to structural Finite Element Models (FEM). The FEM generates distortions of the JWST optical elements. The distortions are then applied to an optical model that solves for the optical performance changes. All these models are subject to the quality of the models, and tests are performed to establish the model's ability to provide accurate metrics. Optical stability feeds back into the overall optical requirement and is dependent on the drift the PM figure (Figure Drift) and the drift of the alignment between the PM and SM (Alignment Drift). For the majority of this paper we will be focusing on the FD metric. FD is defined as the change in wave front error (WFE), measured in nanometers ( $\mathrm{nm}, 10^{-9}$ meters), due to changes in thermal states. From studies and subsystem tests the JWST program has concluded that a Model Uncertainty Factor (MUF) shall be used to increase the structural model predictions by a multiplicative factor of 1.6 for FD.

The structural, thermal and optical models have been in development from the beginning of JWST program. They are some of the largest and most complex models ever developed in support of a NASA mission. Many of the subsystems and smaller components were separately tested and shown to meet system level requirements. However, due to the size and complexity of the JWST telescope, the first and only time it will be thermally tested as a complete assembly is in the Summer 2017. The thermal distortion tests goal will be to demonstrate that the MUF value, 1.6, is conservative when comparing the observed WFE of the PM to the Structural Thermal Optical (STOP) analytical predictions from the models.

NASA performs tests on flight and engineering assembles in order to assure mission success. Testing provides the opportunity to refine, improve and correct models. A common problem with testing is the lack of test response instrumentation when compared to model Degrees of Freedom (DoF). For the upcoming JWST Optical Telescope Element (OTE) and Integrated Science Instrument Module (ISIM), combined as OTIS, Cryogenic vacuum (CV) test there are only 350 temperature sensors, diodes, while the FEM has over 30 million DoF. Therein lies the difficulty in mapping diodes responses to all the structural DoF's. FEMs do not generally provide an accurate method of filling all the models DoF's from such a small thermal input set. Classically this has been done assuming that the temperature information can be assumed to conductively transmit to all the DoF's in the model. FEMs lack thermally related details where a complex thermal model may provide additional heat transfer parameters and variables, such as radiation heat transfer, contact conductance and thermal capacitances. Even the conduction assumption misses the more realistic conduction properties in a thermal model not present in a typical FEM.

As is often the case, the non-flight hardware 'pathfinder' tests were able to accommodate significantly more diodes than are available on the flight hardware OTIS. While the thermal model was off in absolute temperature predictions, the transient trend behaviors in the structure were captured accurately. This finding bolstered the idea that using the thermal model could improve the ability to map a limited diode set to the full FEM DoF set.

The OTIS CV test is a complicated endeavor to test, validate and demonstrate a variety of performance objectives. Two objectives will be a FD and AD test. OTIS will be brought to a stable temperature of 44 Kelvin. While cooling to this stable condition the FD test will be conducted twice. The telescope will change roughly 3 degrees Kelvin in bulk temperature during each FD test. This change will maximize the observable WFE that can be measured with the MultiWave InterFerometer (MWIF) instrumentation. The JWST thermal distortion team will attempt to demonstrate that the MWIF images compare to the STOP analytical predictions to within the FD MUF of 1.6. 
This has led the JWST thermal distortion team to develop a new method of going from limited diode sets to the FEM DoF's using the intelligence of the thermal model to improve the mapping problem. Thermal models have been developed to capture the complex thermal environments and thus gradients within a structure. Using the thermal model properties to improve the process of correlating measured results of an optics performance due to thermal changes. This paper shows how this was achieved and the advantages of such a technique

\section{JWST CRYOGENIC-VACUUM TEST OVERVIEW}

The JWST Pathfinder tests were planned into 3 major test campaigns prior to the final cryo-vacuum test of the fully assembled flight Optical Telescope Elements (OTE) and the Integrated Science Instrument Module (ISIM) (See Figure 2). The three Pathfinder tests are, OGSE1, OGSE2, and the 'Thermal-Pathfinder'. These tests are incremented to be more complex to fully characterize the test facility including the cryogenic test chamber and the supporting ground system equipment (GSE). Furthermore, the 'flight-like' test articles within the Pathfinder tests are designed to understand the thermal behaviors within the cryogenic environment.

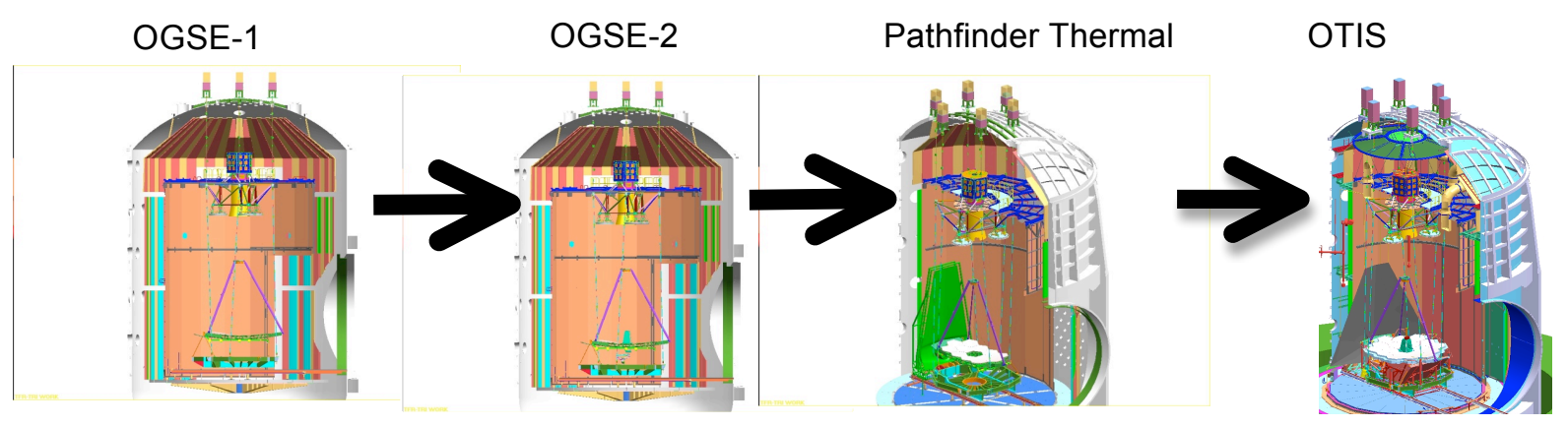

Figure 2. JWST Pathfinder tests are sequentially scheduled into 3 major test campaigns prior to the final cryovacuum test of the fully assembled flight Optical Telescope Elements (OTE) and the Integrated Science Instrument Module (ISIM), combined as called OTIS.

All of the JWST systems-level cryogenic vacuum tests were performed or will be performed at the NASA Johnson Space Center (JSC) Chamber-A (See Figure 3). This chamber was retrofitted with the helium shroud, inboard of the existing liquidnitrogen shroud and it is now capable of providing an environment of less than $20 \mathrm{~K}$. The 30-day OGSE1 test was completed in May 2015 after the JSC Chamber-A was successfully commissioned in 2014 for the JWST use. The OGSE2 cryo-vacuum test was completed in October 2015. The TPF test was completed in October 2016. The final test of the fully assembled JWST Optical Telescope Elements and Integrated Science Instrument Module (OTIS) is currently scheduled to start the Summer of 2017.

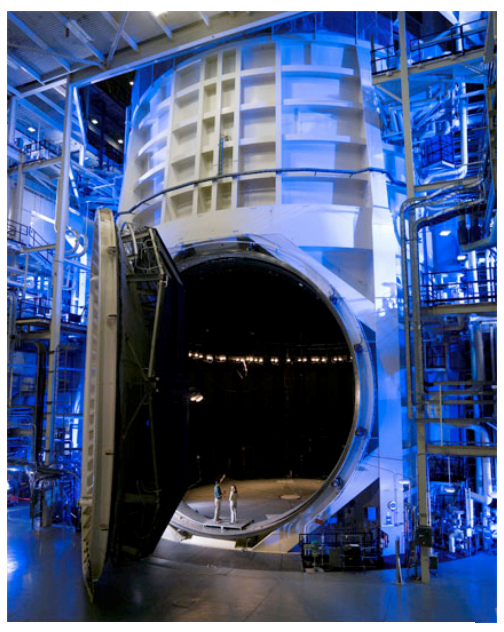

Figure 3 JSC Chamber-A 


\section{APP HEAT DESCRIPTION/PURPOSE/OVERVIEW:}

The thermal output is a set of temperature predictions at discreet locations that are applied as environmental loads to the structural model. Generally speaking, and in the case of JWST, the thermal model used is an entirely different model (mesh density and nodal ID arrangement) than the structural FEM and therefore an intermediate step is necessary to map the thermal loads onto the structural FEM. Ideally you would have a 1:1 ratio between structural and thermal nodes, however, with a thermal model of lower mesh density an extrapolation must occur to fill out the remaining nodes of a structural FEM. For JWST, an 'AppHeat' FEM is created to extrapolate to intermediate nodes which assumes linear conduction between these nodes connected by elements. This method was used for JWST since there was sufficient ratio of thermal to structural nodes to accurately capture the thermal behavior of the structure during the time period of interest.

As the project transitioned from working with a set of thermal model generated predictions to in-test recorded temperatures, the process in which we applied those temperatures to the structural FEM needed to be re-evaluated. In early pathfinder testing it was proven adequate to use the recorded diode temperatures in an AppHeat extrapolation process to populate the structural FEM due to the high density of diodes on the structure and the low error when using these diodes. Diodes were strategically placed on the Unit Under Test (UUT) where the metrics of interest were most sensitive to temperature change. Areas included highly influential composite tubes and near interfaces with other components within the UUT. In addition, other disciplines had placed diodes on areas of the UUT which was also used for the STOP analysis. The resulting error associated with this diode populated AppHeat approach was shown to be acceptable when incorporated with other errors in a test error budget.

Moving forward onto flight hardware OTIS testing resulted in a further reduction of diodes on the UUT. This reduction increased the FEM node to diode ratio, distances to interpolate between seeded nodes on the structural FEM, and the lack of diodes between component interfaces within the UUT. In this scenario the limitations of the AppHeat approach was realized during the TPF test by the team while reviewing overall error in temperature profiles and test metrics as a result of the limited diode set. While the AppHeat approach is still necessary in order to interface with the thermal model, when dealing with a much smaller set of seed/diodes this approach was limited in its effectiveness. 


\section{LDWI DEVELOPMENT}

An observation during TPF test revealed, generally speaking, the test measurements cooled down faster than the pre-test thermal model predictions. In order to characterize the transient thermal model accuracies over the Figure-Drift (FD) phase of the TPF test, thermal nodes mapped/associated with temperature sensing diodes were set to the same temperature as the diode measurement at the start of the FD. Then the same offset was applied to the rest of the transient predictions and the temperature differences were observed over the period from the start of the FD to the end. The observation from this initial comparison revealed that the thermal model predictions may be lacking in the absolute temperature predictions but the transient trend predictions over the FD phase are 'fairly' accurate $(+/-0.2 \mathrm{~K}$ ish over the FD duration of $\sim 2$ days and $2.5 \mathrm{~K}$ Bulk dT of PF. This $\sim 10 \% \mathrm{dT}$ error may be reasonably small as not to cause significant errors in TD predictions due to the relatively constant CTE over that small temperature variation.

Using the stated behavior observed as an assumption, and using the 'sparse' sensor sets and matching thermal model nodes as shown below (see Figure 4), a method was developed in order to 'adjust' the thermal predictions in bulk shift manner. The notional limited sensor considered for this study may represent something even more conservative (ie: fewer sensors) to the sensor set that would be expected at the flight PMBSS-Center Section during the OTIS test. Even though there are a few more temperature sensors on the structure, they were intentionally not considered as a part of the seeded information in order to produce a conservative comparison.

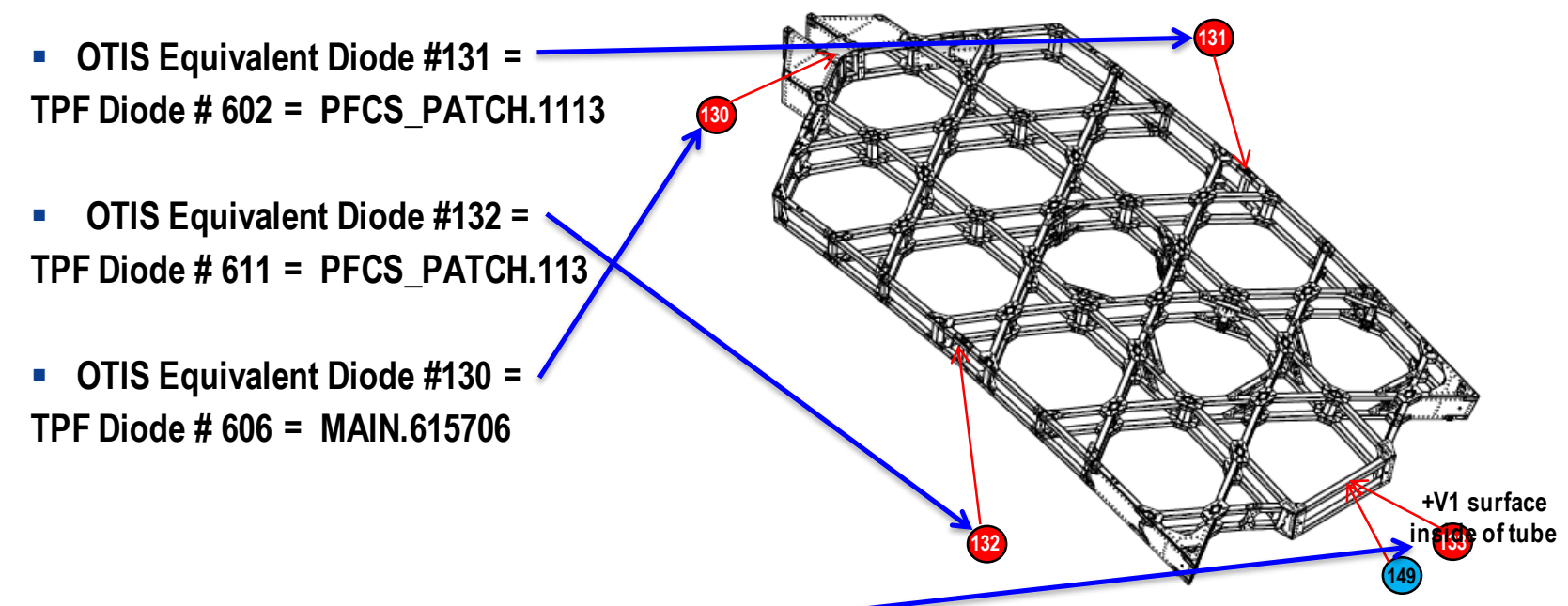

- OTIS Equivalent Diode \#133 =

TPF Diode \# 693 = MAIN.554018

Figure 4 Pathfinder Center Section with OTIS like diode subset 
Shown in Figure 5 and Figure 6 are the complete set of test diodes used during TPF test. There were 171 sensors located on the 'center section' (CS) of the 'pathfinder' composite structure. During TPF test, these test-use-only temperature sensors were populated across the structure in order to capture the temperatures gradients with a higher confidence.

Some of these sensors were placed at very specific locations as these locations were identified with higher sensitivity to the structural distortions when subjected to changes in temperatures. These sensors were used to compare the differences between the model predictions against the measured data during the test.

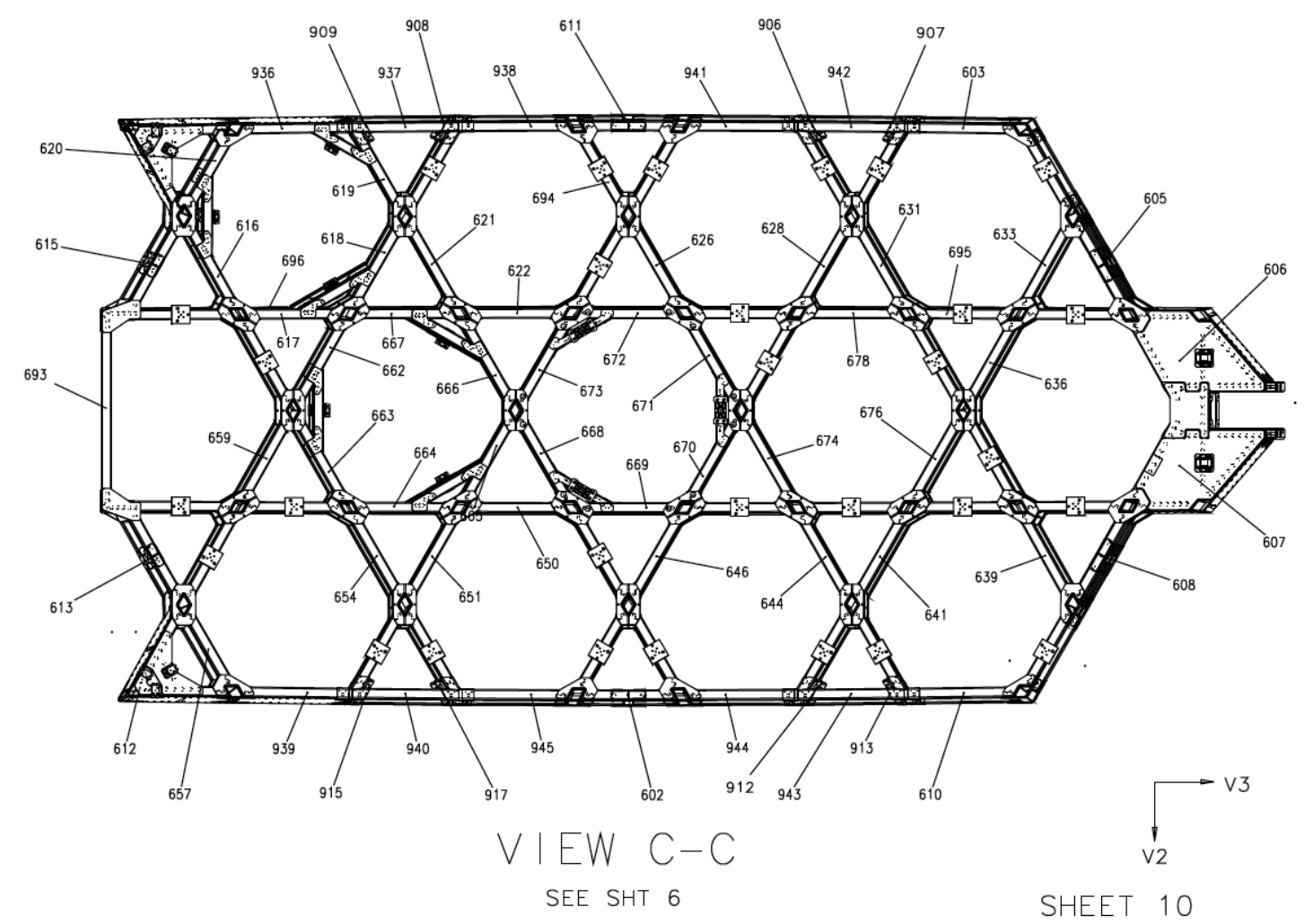

Figure $5+\mathrm{V} 1$ Pathfinder Diode configuration 1 of 2 


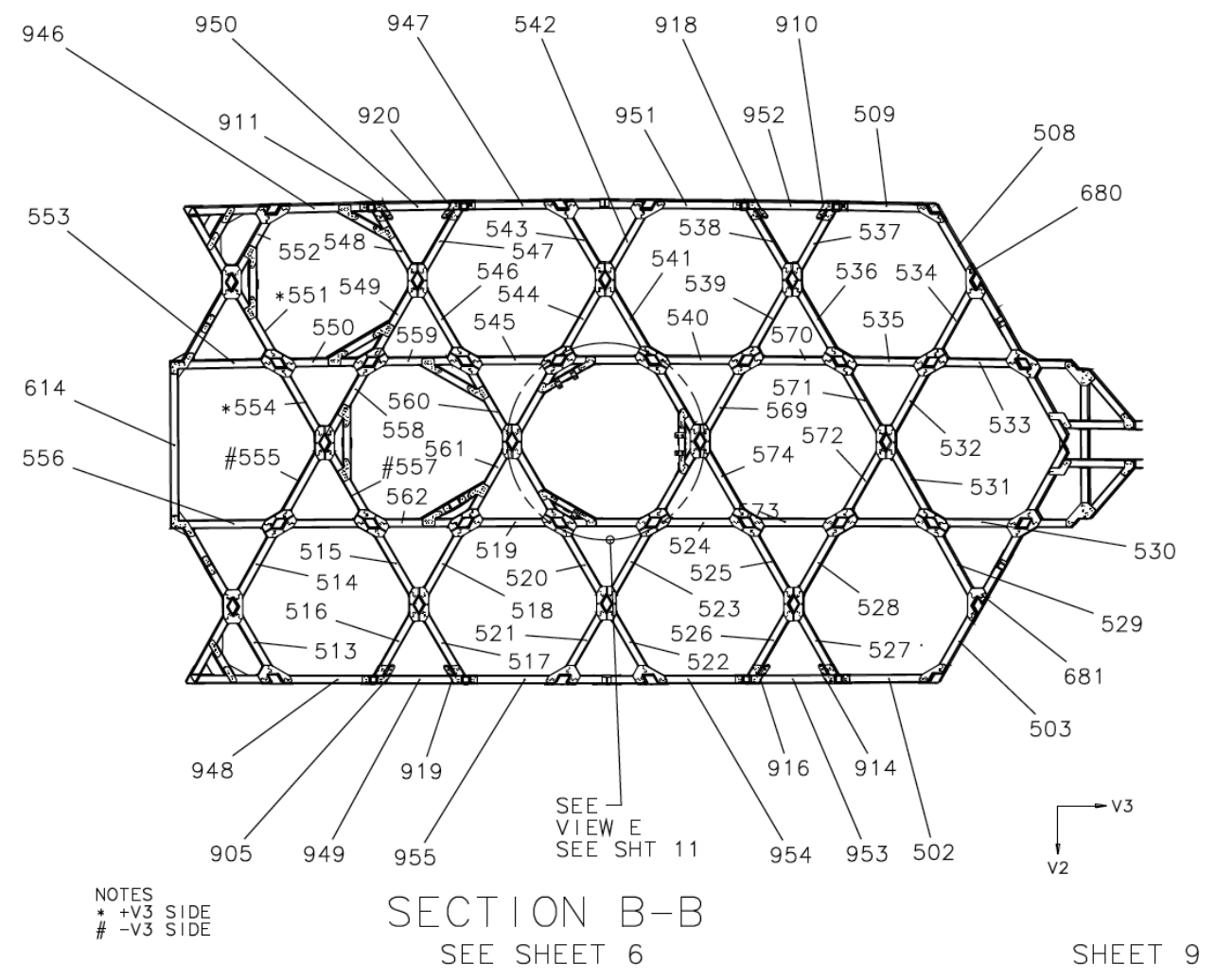

Figure 6 -V1 Pathfinder Diode configuration 2of 2

The following Figure 7 shows the 'raw', unadjusted, differences between the TPF thermal model prediction and the TPF diode measurements of the four notional OTIS PMBSS-CS diode locations. The temperature differences range from $0.56 \mathrm{~K}$ to $8.60 \mathrm{~K}$ depend on their locations.

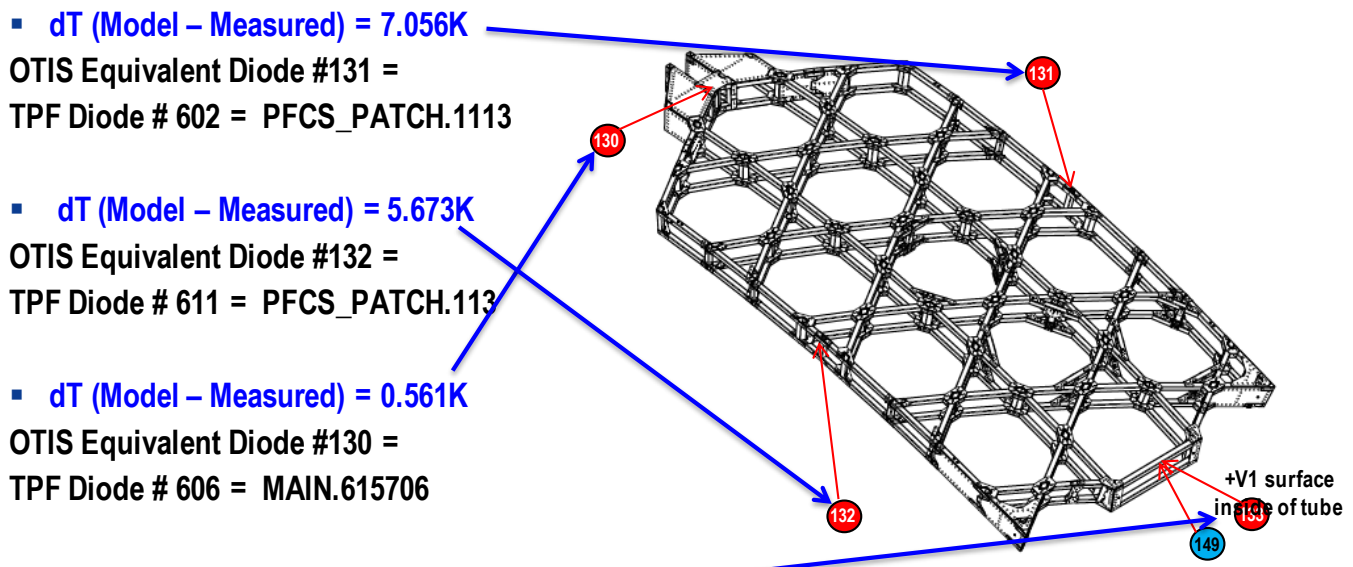

- $\mathrm{dT}$ (Model - Measured) $=8.602 \mathrm{~K}$

\section{OTIS Equivalent Diode \#133 =} TPF Diode \# 693 = MAIN.554018

Figure 7 Thermal model prediction errors during FD at TPF for those selected OTIS like Diode locations 
Shown here on Figure 8 are the unadjusted temperature differences between the thermal model prediction and measured diode data at the start of the FD test $\mathrm{dT}=$ (Model - Measured). The temperature differences among the 167 (171 less 4 seeded sensors) sensors ranged between $0.34 \mathrm{~K}$ and $9.46 \mathrm{~K}$, mostly less than approximately $7 \mathrm{~K}$, with a linear average dT of $5.62 \mathrm{~K}$.

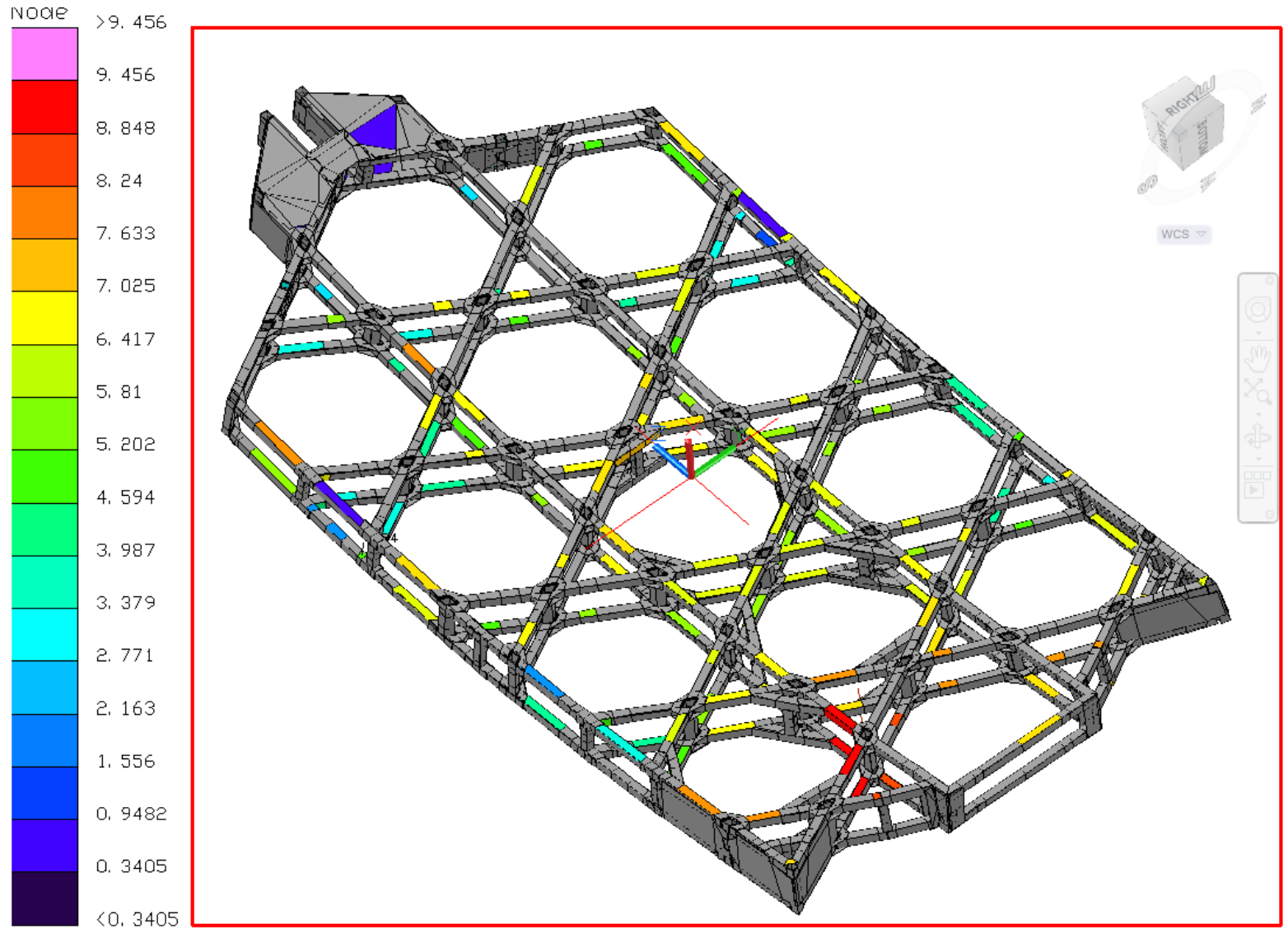

TPF_CS_UnAdjusted_Predictions_113016. txt

IPF CS UnAdiusted Predictions 113016. txt

Figure 8 Thermal model prediction errors during FD at TPF 
The Linear Distance Weighted Interpolation (LDWI) method was developed to augment the thermal model predictions based on the sparse sensor information. As an example, using the TPF diode location 502, Model MAIN.553531 (see Figure 9), the nodal prediction of MAIN.553531 would be adjusted by the notional 4 seed-diodes by the inverse linear distance relationships (ie: closer to the seed diode, the higher the influence on the adjusted temperatures).

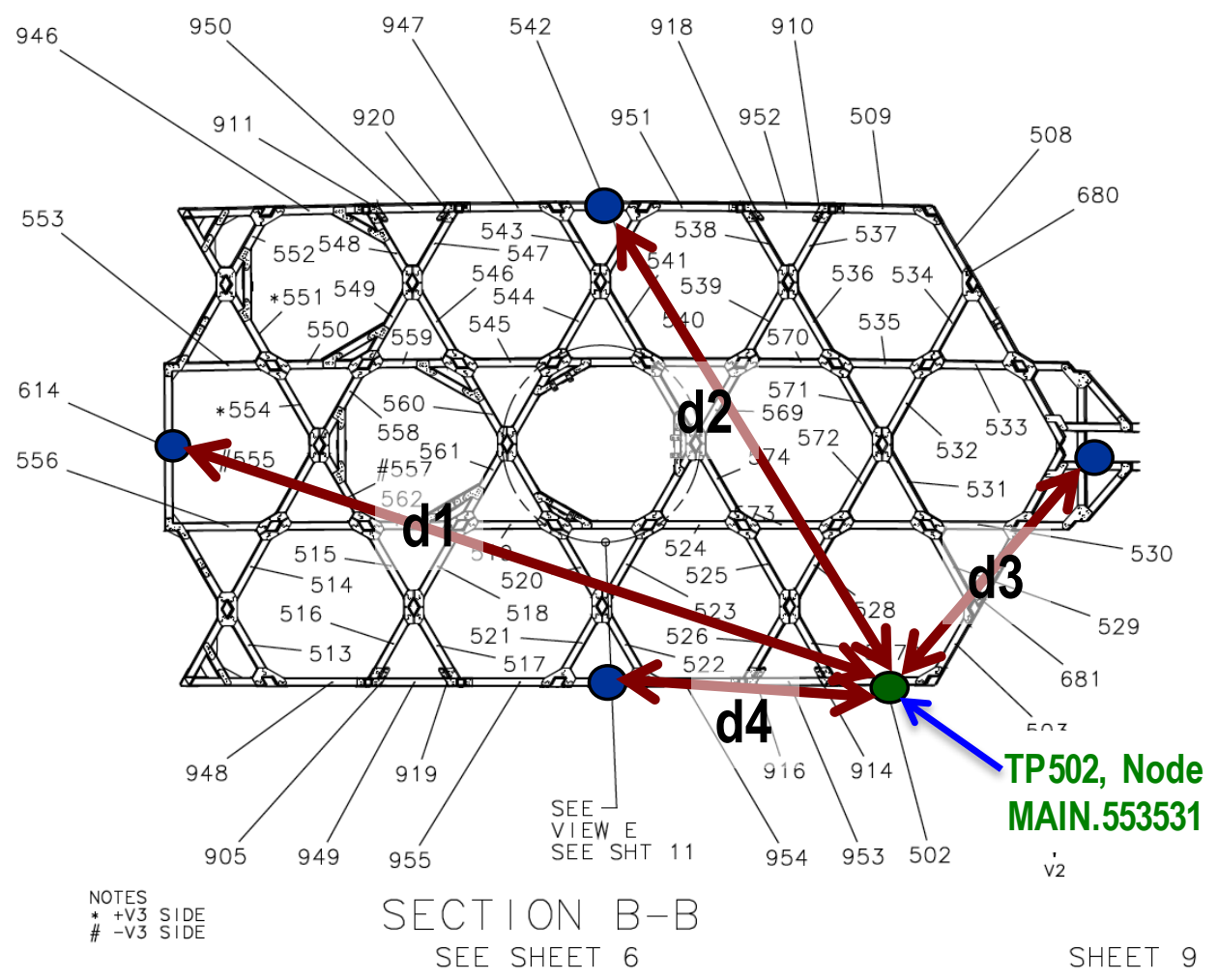

Figure 9 Example LDWI processing

The MAIN.553531 prediction 'adjustment' could be made using the following relationship equation:

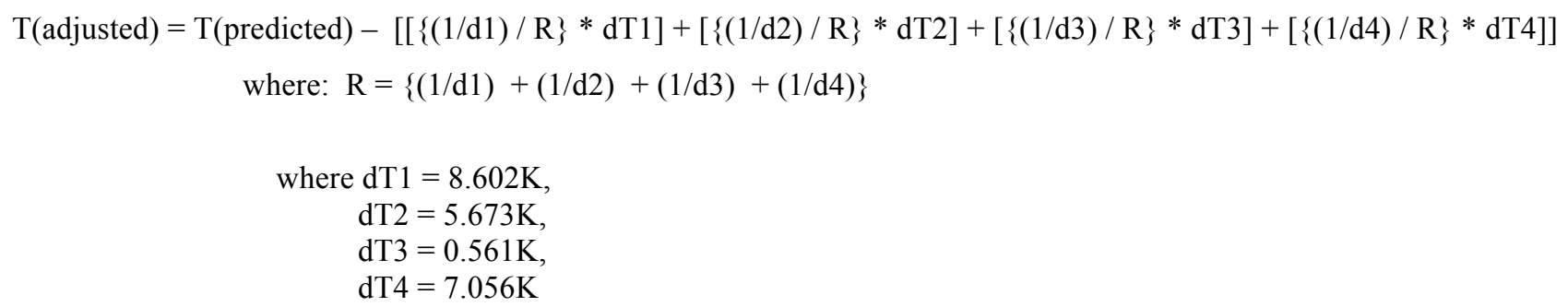

Shown in Figure 10 are the ADJUSTED temperature differences between the model prediction and measured diode data at the start of the FD test dT $=$ (Model - Measured). The temperature differences among the 167 (171 less 4 seeded sensors) sensors 'mostly' below $\sim 3 \mathrm{~K}$ (was $7 \mathrm{~K}$ ), with a linear average dT of $1.85 \mathrm{~K}$ (was $5.62 \mathrm{~K}$ ). 


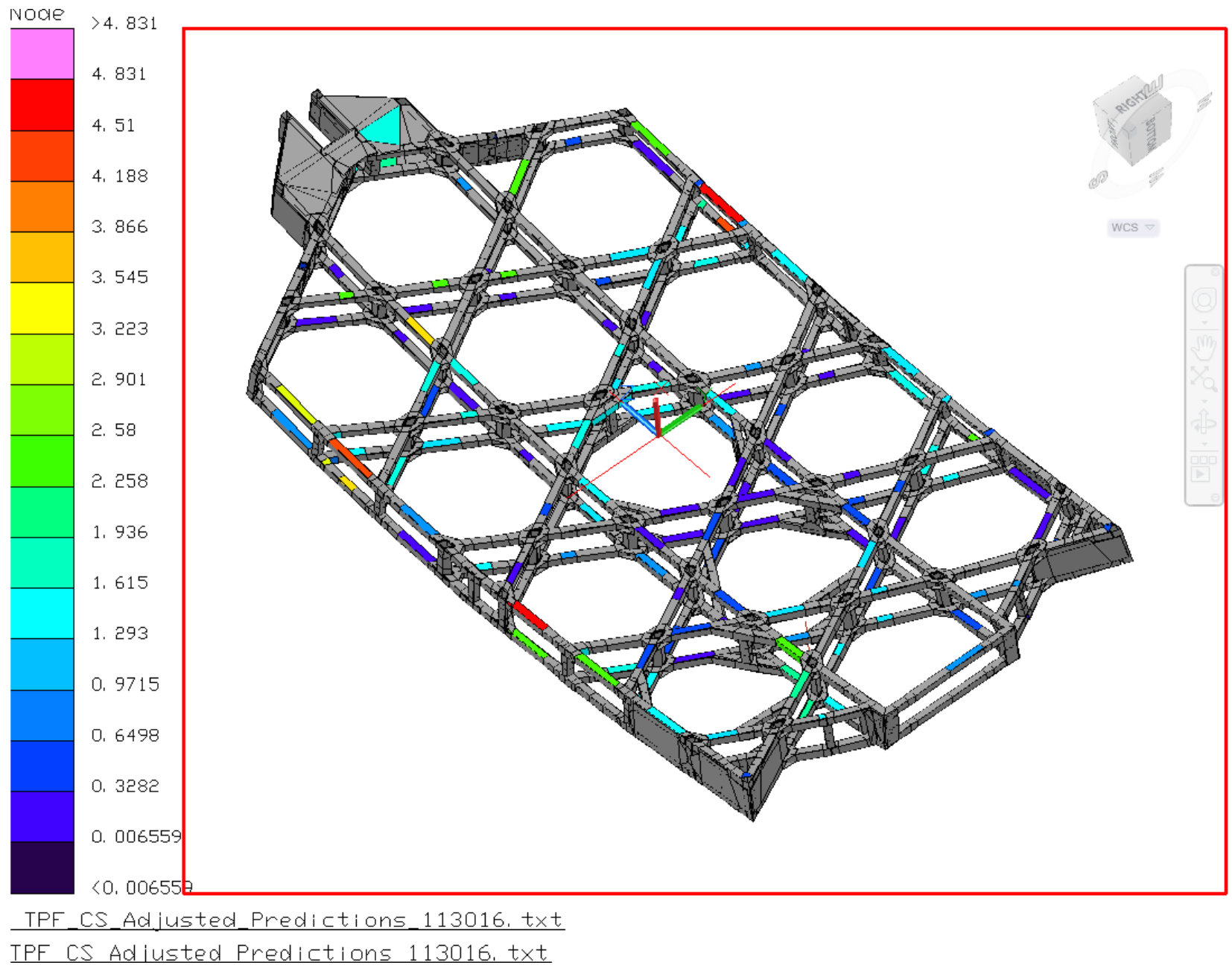

Figure 10 LDWI adjusted thermal modeling errors at TPF

\section{EVALUATING EFFECTIVENESS OF MAPPING PROCESSES}

In order to evaluate the effectiveness of different approaches to mapping the structural FEM, we first start with a baseline. This baseline is also referred to as the gold standard. The Gold Standard (GS) set originated from a full thermal node set that was used in the AppHeat approach to extrapolate out to all structural nodes. From here delta plots can be created in order to evaluate effectiveness of the proposed mapping process. Figure 11 is an example delta temperature plot created from a full set of thermal model nodes extrapolated over the PMBSS CS and Wings using the AppHeat method. Figure 12 starts with the same set of thermal predictions, however, instead of using the entire node set for the AppHeat process, only a few set of nodes that correspond to diode locations on the flight hardware are used. Figure 13 uses the LDWI method for mapping the structural FEM based on the same limited OTIS set of diodes. 

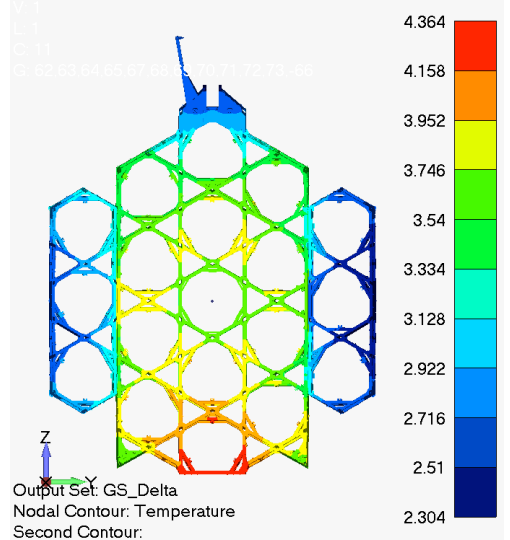

Figure 11 dT GS Temperature Plot

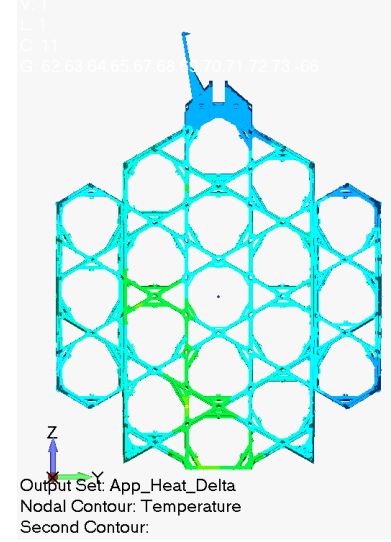

Figure 12 dT OTIS AppHeat Temperature Plot
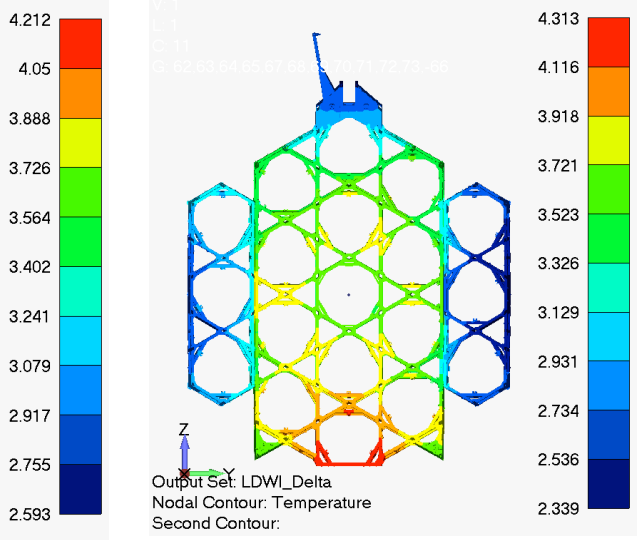

Figure 13 dT LDWI Temperature Plot

Another way to evaluate the above plots is to subtract the proporsed methods from the GS plot. Effectively creating a temperature mapping error plot (Figure 15 \& Figure 14). This qualitative approach shows the LDWI approach is much better at capturing gradients across the PMBSS structure.

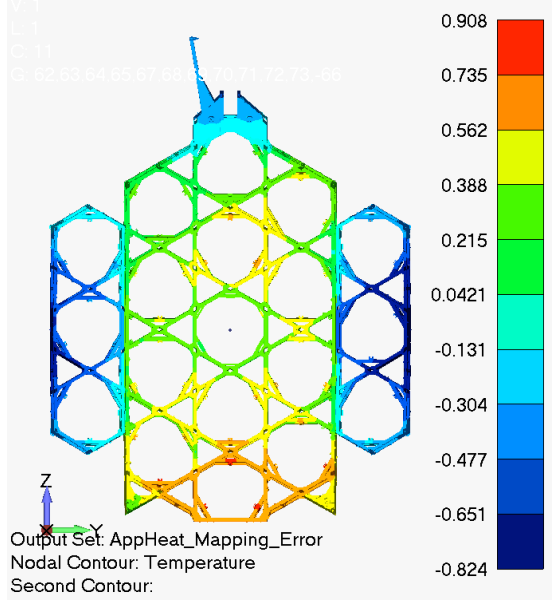

Figure 15 dT Thermal Mapping error plot AppHeat

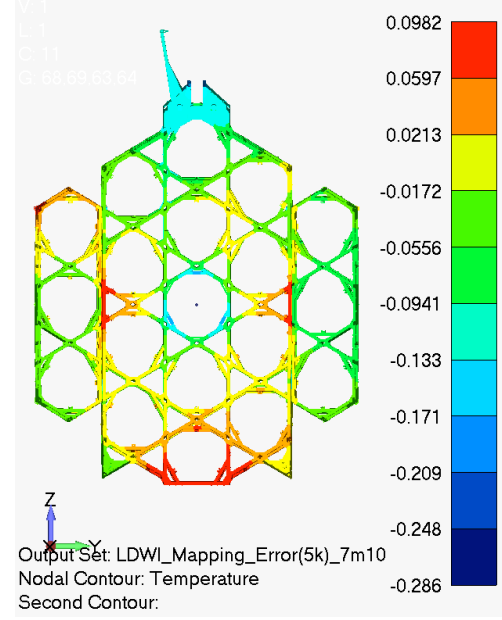

Figure 14 dT Thermal Mapping error plot LDWI

For a quantitative approach of evaluating the effectiveness of the mapping approaches is to examine test metrics for each of the methods compared to the GS. For this purpose we look at the delta RMS WFE metric between the GS and each of the mapping approaches. As seen in Figure 16 and Figure 17, the AppHeat approach produced roughly eight times more error in the RMS WFE metric over the LDWI approach for this thermal case. 


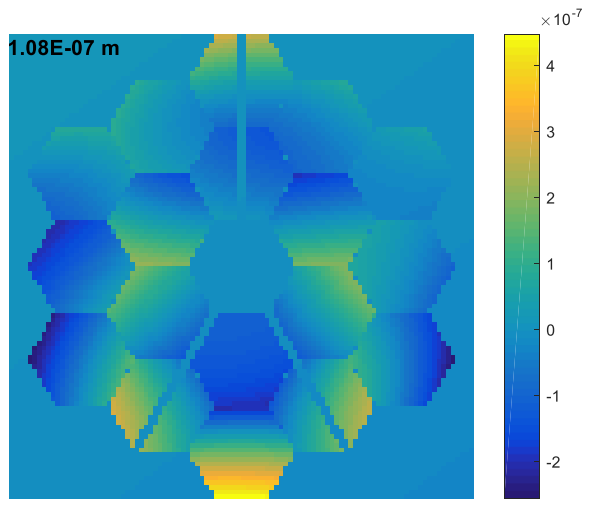

Figure 16 AppHeat method mapping error $(108 \mathrm{~nm}$ RMS)

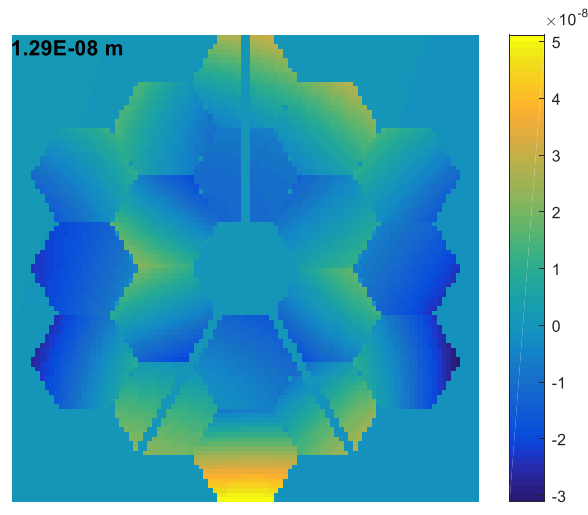

Figure 17 LDWI method mapping error (13 nm RMS)

\section{CONCLUSIONS}

STOP analysis is a critical part of the design, construction, and testing of any space telescope. Having seamless integration between thermal, structural and optical modeling/analysis is key for the STOP analysis to be effective. The limited sensor counts cause shortfalls of a thorough understanding of the temperature distributions in the OTIS PMBSS during the OTIS transitions (ie: cool-down and warm-up, more specifically to FD and AD phases). Leading up to OTIS testing thorough pathfinder testing let the team gain confidence in their models, analysis, and processes that would play a major role in final OTIS testing. From pathfinder testing the team was able to understand the thermal behavior of an OTIS like PMBSS structure in an OTIS like cooldown timeline and environment. From this the team was able to develop a new method (LDWI) for populating a structural FEM with limited in-test thermal diode data and asset of thermal modeling predictions. Another advantage of the LDWI method is that it may be executed near real time during the OTIS FD and AD tests. The thermal model run times are currently very lengthy and may not be suitable for in-situ test analysis to support the TD tests. Assuming that the LDWI method may provide better thermal data to FEM, the thermal model would go through a post-test 'model' improvement, such as adjusting for measured boundary conditions but not necessarily a full-up thermal model correlation. Then, this post-test improved thermal model may provide even better thermal nodal predictions contributing towards the success of JWST thermal distortion tests by using the LDWI method. 